\title{
Improved Bush-Based Methods for Network Contraction
}

\author{
Ehsan Jafari ${ }^{1, *}$, Stephen D. Boyles ${ }^{1}$ \\ ${ }^{1}$ Department of Civil, Architectural and Environmental Engineering, The University of Texas at Austin
}

\begin{abstract}
Calculating equilibrium sensitivity on a bush can be done very efficiently, and serve as the basis for a network contraction procedure. The contracted network (a simplified network with a few nodes and links) approximates the behavior of the full network but with less complexity. The network contraction method can be advantageous in network design applications where many equilibrium problems must be solved for different design scenarios. The network contraction procedure can also be used to increase the accuracy of subnetwork analysis. This method requires calculating travel time derivatives between two nodes, with respect to the demand between them, assuming that the flow distributes in a way that equilibrium is maintained. Previous research describes two methods for calculating these derivatives. This paper presents a third method, which is simpler, faster, and just as accurate. The method presented in this paper reformulates the linear system of equations defining these sensitivities as the solution to a convex programming problem, which can be solved by making minor modifications to static user equilibrium algorithms. In addition, the model is extended to capture the interactions between the path travel times and network flows, and a heuristic is proposed to compute these interactions. The accuracy and complexity of the proposed methodology are evaluated using the network of Barcelona, Spain. Further, numerical experiments on the Austin, Texas regional network validate its performance for subnetwork analysis applications.
\end{abstract}

Keywords - Network contraction; Equilibrium bushes; Bush sensitivity analysis; Network Interactions; Subnetwork analysis.

\section{Introduction}

Static traffic assignment remains the most common network equilibrium model in practice: its favorable mathematical properties are well-known, practitioners are experienced at calibrating and interpreting the results, and it can be solved quickly. In particular, algorithms based on bushes - a concept dating to Dial (1970), and first applied to the static equilibrium problem in Dial (1999b) — exploit the acyclicity of the equilibrium flows to solve for equilibrium rapidly.

An important consequence of Beckmann et al.'s formulation (1956) is that a meaningful sensitivity analysis can be undertaken. In the context of network equilibria, sensitivity analysis refers to determining a functional relationship between the travel times and demands without re-solving the network equilibrium problem. This functional relationship can be used to represent the network by connecting each origindestination (OD) pair with a single artificial link and removing all the intermediate nodes and links.

*Corresponding author.

E-mail addresses: ejafari@utexas.edu (E. Jafari), sboyles@mail.utexas.edu (S.D. Boyles). 
The main reason to develop such network contraction techniques is reducing the computational burden of solving many network equilibrium problems (Friesz, 1985). Still this computational motivation seems to be valid. As an example, consider a network design problem which is formulated as a bi-level problem. The master problem deals with computing some design parameters, and in the equilibrium subproblem, the travelers modify their route choice in response to design parameters set in the master problem. Such problems require solving hundreds or thousands of the network equilibrium problem as a subproblem which can be computationally expensive even for modern algorithms such as Algorithm B (Dial, 2006), TAPAS (Bar-Gera, 2010), or LUCE (Gentile, 2014).

As another application, consider the case of studying a number of alternatives, such as a new signal timing plan or converting a one-way local street to two ways, in a region of interest. The impacts of these policies are expected to be local, and solving the whole regional network may be unnecessary. Subnetwork analysis is commonly used to avoid incurring the computational burden of regional modeling. In practice, subnetwork modeling usually involves extracting a small component of a regional network, allowing the boundary nodes of the subnetwork to serve as origins and destinations, and estimating the subnetwork trip table from an equilibrium solution on the regional network. Xie et al. (2010) use entropy-maximization to identify these trips between the boundary points of the subnetwork. Effectively, this forms a "fixed" boundary condition which neglects diversion effects due to changes in the subnetwork.

Boyles (2012) adopts a different approach, using bush-based sensitivity analysis to simplify the regional network, rather than delete it entirely. In this way, diverting flows can be approximated while still retaining most of the computational advantage of subnetwork modeling; the boundary is less rigidly enforced. The chief advantage of this procedure is that it captures diversion in a behavioral manner, based on drivers choosing routes to minimize their travel time. This contrasts with the fixed-boundary approach, where a common question is "how large does the subnetwork need to be to capture diversion?" While natural, this question is a bit of a red herring. Consider a downtown area served by two roughly parallel freeways (Figure 1). Even at large distances from the downtown area, there are drivers whose origins are roughly equidistant from these two freeways. These drivers' choice between these freeways depends on travel patterns downtown, regardless of how distant their origin is: the fundamental issue is modeling route choice, not simply capturing a large enough area. By integrating one model into another, "smoothing" the boundary, much faster convergence and greater accuracy can be seen without needing a large subnetwork.

Creating such a model requires estimation of a number of sensitivity parameters. Boyles (2012) provided two methods to calculate these parameters: one is reminiscent of resistive circuit analysis, and only applies when the equilibrium bushes are planar. The other is based on iterative solution of a linear system, exploiting the underlying network structure to avoid inverting any large matrices. Both of these methods, however, have undesirable aspects. In modern regional networks, planar bushes are rare because freeway interchanges and overpasses are modeled in detail, rather than representing the entire interchange with a single node (as was done in past decades to reduce the number of nodes and arcs). For example, in the Chicago regional network (Bar-Gera, 2013), none of the 1790 origin bushes are planar at equilibrium. While the second method is applicable in general networks, it requires careful implementation to avoid numerical instabilities due to repeated matrix reinversion. 


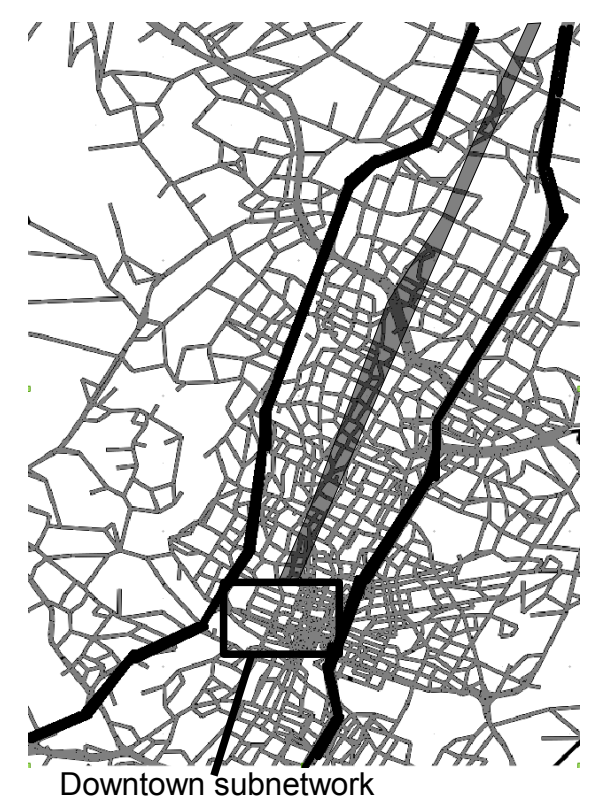

Figure 1: Portion of regional network with equidistant region shaded. Route choice from this region to downtown is sensitive to changes in the subnetwork regardless of distance between the origin and downtown.

Also, these previous methods assume that the travel time between the origin and destination nodes of each OD pair is a function of its own demand, and independent of other OD demands. In case of a congested network, this assumption may degrade the accuracy of the method. Due to overlapping paths, a small change to the flow on one path affects the travel time experienced by other paths. The set of impacted paths and OD pairs extends further when some travelers decide to reroute to other paths in response to travel time increase on their current path. In this paper, the formulation is revised to model these interactions: the dependency of each OD travel time on the demand of other OD pairs. In fact, modeling OD travel time as a function of its demand can be viewed as a special case of the model proposed in this paper, and further a heuristic is proposed to address the question of which OD pairs should be included in modeling the travel time of each OD pair.

In addition, this paper describes a third method for calculating the necessary parameters, by reformulating the linear system of equations as the solution to a modified network equilibrium problem. This method is faster than the previous methods, makes no planarity assumptions, and can be solved by making only minor adjustments to existing equilibrium algorithms. In summary, the contributions of this paper are as follows:

- The contracted model is extended to include the interactions between different OD pairs.

- A symmetry result is found in the sensitivity of travel times and demands across different OD pairs: the impact of increased OD flow on another OD pair's cost is the same as the impact of increased flow of the second OD pair on the first OD pair's cost.

- A heuristic is proposed to approximate the most prominent interactions.

- A formulation similar to the network equilibrium problem is developed to carry out the sensitivity 
analysis and compute the derivatives.

The remainder of the paper is organized as follows: Section 2 discusses related research on network contraction, sensitivity analysis, and bush-based algorithms. Section 3 briefly reviews the context and objectives of the problem at hand, and Section 4 presents the novel method based on reformulation as an equilibrium problem. Section 5 describes a heuristic for estimating the interactions. Section 6 demonstrates the accuracy and computational performance of this procedure using two case studies. The Barcelona network is used to measure the complexity and to evaluate the accuracy of the proposed model in response to different demand perturbations. The advantages of simplifying the regional network rather than deleting it in subnetwork analysis is illustrated on a regional network representing Austin, TX. Section 7 concludes the paper.

\section{Literature Review}

The idea of simplifying networks to reduce computation time has existed for several decades, receiving considerable attention prior to the rise of efficient path-based and bush-based equilibrium algorithms since the 1990's. In case of an uncongested network, networks with constant link travel cost, the problem is easy to handle by using aggregated nodes instead of a group of the network nodes. Zipkin (1980) derives bounds for such a contraction technique for a class of linear minimum cost flow problems. As noted by Friesz (1985), the network contraction problem becomes complicated and computationally intensive for congested networks.

Different contraction methods have been studied in the presence of congestion. The early studies on network contraction used the idea of link extractions (Haghani and Daskin, 1983). Link extraction refers to removing unimportant links and nodes from the network. Chan (1976) proposes such a network contraction approach for networks with constant link cost. This study shows that the derived traffic from the excluded links cause an unpredictable flow pattern through the network. Hearn (1984) proposes an analytical method for link extraction where the subnetwork of interest is extracted and then a transfer decomposition is applied to partition the original traffic assignment problem into a master problem and a subproblem. The master problem deals with a modified version of the original network where the subnetwork is replaced with some pseudo-links, and the subproblem solves the traffic assignment problem over the extracted subnetwork.

Some efforts have been done to combine the link extraction with demand aggregation. Modeling the transportation network with full demand resolution requires detailed data which is expensive and time consuming. Zonal aggregation can reduce the computational expense of detailed zoning by dividing the space into discrete zones called traffic analysis zones. It is assumed that zone activity is concentrated at the zone centroid and demand are loaded on and of the network through connectors (Jafari et al., 2015). By evaluating two levels of network details and 11 zoning structures, Chang et al. (2002) show that smaller zones are more beneficial in travel demand modeling, while larger zones result in a better performance for less detailed network. Jeon et al. (2010) construct three networks with different levels of contraction: a fine, a medium (collectors are excluded), and a coarse model (collectors and minor streets are deleted). Zones are also aggregated based on the method proposed by Bovy and Jansen (1983). The simulation results show that the reduced capacity is hard to estimate, and traffic volumes are overestimated on the remaining links as a 
result of this reduced capacity. Also studies by Eash et al. (1983) and Sbayti et al. (2002) show that due to inconsistency between the extracted network and the complete network, the results from these methods are not reliable.

Another contraction approach is link abstraction where a set of links and nodes between two nodes are replaced with a single aggregated link. Eash et al. (1983) and Boyce et al. (1985) propose rules to aggregate series and parallel links for the purpose of the sketch planning. This idea is used by Wright et al. (2010) to reduce the computational time and increase the efficiency of the algorithm for large-scale networks. Connors and Watling (2014) employ the idea of link abstraction for stochastic user equilibrium (SUE) problem where the route choice is a function of the path utility. In SUE there is no notion of equilibrium travel time, and the authors propose the composite cost as a unique measure for each OD pair. Similar to Boyles (2012), sensitivity analysis is used to model the OD composite cost as function of the OD demands.

The methodology proposed in this paper can be classified as link abstraction method based on the equilibrium sensitivity analysis and is a sequel to work done by Boyles (2012). Explicit sensitivity analysis for the static equilibrium problem has primarily been based on the implicit function theorem (Tobin and Friesz, 1988; Cho et al., 2000; Yang and Bell, 2007) or sensitivity results of variational inequalities (Lu, 2008). Recently, Bar-Gera et al. (2013) formulated the sensitivity of link flows with respect to network design parameters as a solution to a quadratic programming and evaluated the precision of the computed derivatives. Other researchers have established general regularity properties on the solution of network equilibrium problems as the input data vary, identifying conditions under which the equilibrium solution (expressed either in terms of link or path flows) is continuous or differentiable (Qiu and Magnanti, 1989; Yen, 1995; Patriksson, 2004; Lu and Nie, 2010). Particularly relevant to this problem, equilibrium bushes are stable and link flows are analytic with respect to demand perturbations under generally nonrestrictive regularity assumptions (Boyles, 2012).

In static traffic assignment, there is always an equilibrium flow solution in which the links used by each origin form an acyclic subgraph (Bar-Gera, 2002). Due to the existence of a topological order (Ahuja et al., 1993), network algorithms on acyclic graphs are usually very fast. To the author's knowledge, this notion

of a bush (an acyclic subgraph) dates to Dial (1970), where it was applied in a stochastic network loading procedure. The same concept was used in a network pricing problem (Dial, 1999a), and independently rediscovered in the setting of communications networks (Gallager, 1977), before being applied to the static equilibrium problem as the basis for a number of algorithms (Dial, 1999b; Bar-Gera, 2002; Dial, 2006; Nie, 2010; Gentile, 2014).

\section{Problem Statement}

Consider a directed network $G=(N, A, Z)$ with node and arc sets $N$ and $A$ of cardinality $n$ and $m$, respectively, and a set of zones $Z \subseteq N$ of cardinality $z$. Let $W \subseteq Z \times Z$ denote the set of $K$ OD pairs. The travel demand between OD pair $w$ is indicated by $d_{w}$. We group the OD demands into a $K$-dimensional vector $\mathbf{d}$. Let $p_{w}$ denote the set of all paths connecting OD pair $w$ and $\mathbf{p}$ be the entire set of paths in the network. The delay on each link $a$ is given by $t_{a}\left(x_{a}\right)$, as a function of its flow $x_{a}$ which is strictly positive, 
Table 1: Table of Notation

\begin{tabular}{|c|c|c|}
\hline$N$ & $\triangleq$ & set of network nodes of cardinality $n$ \\
\hline$A$ & $\triangleq$ & set of network links of cardinality $m$ \\
\hline$Z$ & $\triangleq$ & set of network zones of cardinality $z$ \\
\hline$W$ & $\triangleq$ & set of network OD pairs of cardinality $K$ \\
\hline$w \in W$ & $\triangleq$ & an OD pair \\
\hline $\mathbf{d}=\left[d_{w}\right]$ & $\triangleq$ & vector of OD demands \\
\hline $\mathbf{p}=\left[p_{w}\right]$ & $\triangleq$ & vector of OD paths \\
\hline$\hat{p}_{w}$ & $\triangleq$ & set of paths with positive flow between OD pair $w$ \\
\hline$T_{w}$ & $\triangleq$ & travel time between OD pair $w$ \\
\hline$h_{\pi}$ & $\triangleq$ & flow of path $\pi$ \\
\hline$C_{\pi}$ & $\triangleq$ & cost of path $\pi$ \\
\hline$x_{a}$ & $\triangleq$ & flow on link $a$ \\
\hline$t_{a}\left(x_{a}\right)$ & $\triangleq$ & cost of traveling on link $a$ \\
\hline$\delta_{a \pi}$ & $\triangleq$ & 1 if link $a$ is on path $\pi$, otherwise 0 \\
\hline $\mathbb{B}_{r}$ & $\triangleq$ & equilibrium bush rooted at origin $r$ \\
\hline $\mathbb{N}_{r}$ & $\triangleq$ & set of nodes visited by some demand from origin $r$ \\
\hline $\mathbb{A}_{r}$ & $\triangleq$ & set of links visited by some demand from origin $r$ \\
\hline $\mathcal{B}_{w}$ & $\triangleq$ & stem of OD pair $w$ \\
\hline $\mathcal{N}_{w}$ & $\triangleq$ & nodes utilized by $d_{w}$ \\
\hline $\mathcal{A}_{w}$ & $\triangleq$ & links utilized by $d_{w}$ \\
\hline$N_{c}$ & $\triangleq$ & set of nodes in contracted network \\
\hline$A_{c}$ & $\triangleq$ & set of artificial links in contracted network \\
\hline$Z_{c}$ & $\triangleq$ & set of zones in contracted network \\
\hline$l_{w}$ & $\triangleq$ & artificial link between OD pair $w$ in contracted network \\
\hline$\Upsilon_{w}$ & $\triangleq$ & cost on artificial link between OD pair $w$ \\
\hline$G(w)$ & $\triangleq$ & $\begin{array}{l}\text { set of OD pairs selected to model the travel time between origin } \\
\text { and destination nodes of } w\end{array}$ \\
\hline$\alpha_{a}^{w}$ & $\triangleq$ & derivative of $x_{a}$ with respect to $d_{w}$ \\
\hline$\beta_{\pi}^{w}$ & $\triangleq$ & derivative of $h_{\pi}$ with respect to $d_{w}$ \\
\hline$\tau_{u}^{w}$ & $\triangleq$ & derivative of $T_{u}$ with respect to $d_{w}$ \\
\hline$O D_{a}$ & $\triangleq$ & set of OD pairs with demand on link $a$ \\
\hline
\end{tabular}


strictly increasing, and differentiable. The deterministic user equilibrium problem seeks the vector of link flows $\hat{\mathbf{x}}$ minimizing the following convex optimization problem:

$$
\begin{array}{lll}
\text { minimize } & \sum_{a \in A} \int_{0}^{x_{a}} t_{a}(x) d x & \\
\text { subject to } & \sum_{\pi \in p_{w}} h_{\pi}=d_{w}, & \forall w \in W \\
& \sum_{w \in W} \sum_{\pi \in p_{w}} h_{\pi} \delta_{a \pi}=x_{a}, \quad \forall a \in A \\
& h_{\pi} \geq 0, & \pi \in \mathbf{p}
\end{array}
$$

where $h_{\pi}$ is the flow on path $\pi$, and $\delta_{a \pi}$ is 1 if path $\pi$ utilizes link $a$, and 0 otherwise. Assume that the unique link flow vector $\hat{\mathbf{x}}$ satisfying the above problem is known, and let $\hat{T}_{w}$ be the minimum travel time between OD pair $w$ at equilibrium flow $\hat{\mathbf{x}}$. Furthermore, let $\hat{p}_{w}$ denote the set of paths with positive flow for $w$ corresponding to $\hat{\mathbf{x}}$. These might represent the entropy-maximizing path flows, or some other path-based solution. The route flow is assumed to be strictly complementary in the sense that all minimum-cost routes have positive flow. This assumption is common in the sensitivity analysis literature (Friesz et al., 1990; Cho et al., 2000; Patriksson, 2004; Josefsson and Patriksson, 2007; Yang and Bell, 2007). In practice, it can be difficult to determine whether a solution violates strict complementarity, because equilibria are only solved to finite precision (cf. Bar-Gera (2006)), but noncomplementary solutions are a zero-measure set (Patriksson, 2004) so we feel this assumption is viable for practical applications such as the ones considered in this paper.

The equilibrium flow from each origin to all destinations form a bush, a connected and acyclic subnetwork connecting origin to each node. Define the equilibrium bush rooted at origin $r$ to be $\mathbb{B}_{r}=\left(\mathbb{N}_{r}, \mathbb{A}_{r}\right)$, where $\mathbb{N}_{r} \subseteq N$ and $\mathbb{A}_{r} \subseteq A$, respectively, are sets of nodes and links visited by some demand from $r$. For each OD pair $w \in W$ with origin $r$, stem $\mathcal{B}_{w}=\left(\mathcal{N}_{w}, \mathcal{A}_{w}\right)$ is defined as a subgraph of equilibrium bush $\mathbb{B}_{r}$ comprised of links and nodes utilized by $d_{w}$ at equilibrium flow $\hat{\mathbf{x}} ; \mathcal{A}_{w}$ and $\mathcal{N}_{w}$ are stem links and nodes, respectively. More precisely, the stem $\mathcal{B}_{w}$ is a subnetwork formed by the union of links and nodes visited by at least one path in $\hat{p}_{w}$.

We seek to estimate a simple equation to model the relation between the OD travel time and network flows. By doing so, network $G$ is replaced by a "contracted" network $G_{c}=\left(N_{c}, A_{c}, Z_{c}\right)$ with the following properties: $(a)$ the contracted network has the same set of zones as the original network and does not contain any intermediate node, i.e. $N_{c}=Z_{c}=Z$; $(b)$ the number of links in the contracted network is the same as number of OD pairs in $G$. More precisely, there is one artificial link between each OD pair in the contracted network. If network $G$ has $n$ nodes, $m$ links, $z$ zones, and $K$ OD pairs, the contracted version includes $z$ nodes, $z$ zones and $K$ links. Figure 2 shows this transformation on a small network. The complete network on the left panel has 1 origin node, node 1 , and 2 destination nodes, nodes 3 and 5 , while the associated contracted network, right panel, has just 3 nodes and 2 links; one node for each origin/destination node and one artificial link between each OD pair. 

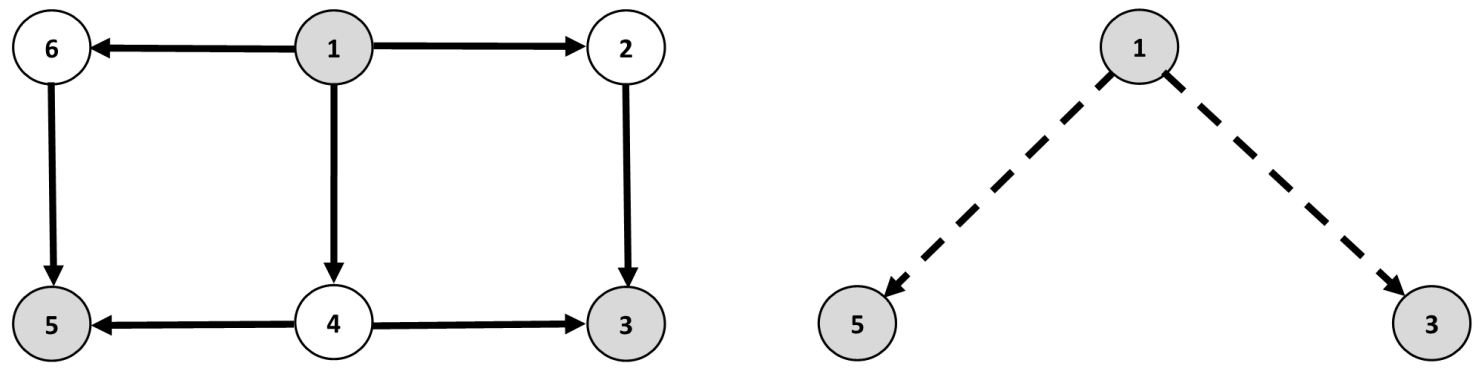

Figure 2: The complete network (left) has 6 nodes and 7 links, while the contracted network (right) has just 3 nodes representing the zones, and 2 artificial links representing each OD pair.

The artificial links in the contracted network model the travel time between each OD pair as a function of OD flows. Let $l_{w}$ denote the artificial link between OD pair $w$ in the contracted network and $\Upsilon_{w}$ denote the travel time on $l_{w}$. As discussed before, $\Upsilon_{w}$ estimates the cost of travel between OD pair $w$ in the complete network $G$. Boyles (2012) assumed that $\Upsilon_{w}$ is a function of $d_{w}$ alone, and independent of other OD demands, i.e. $\Upsilon_{w}=\Upsilon_{w}\left(d_{w}\right)$. In this paper, however, this assumption is relaxed, and the cost of travel on each artificial link is formulated as a multivariable function to capture the interactions between multiple OD flows.

Using $\hat{\mathbf{d}}$ to denote the demand vector at the equilibrium solution $\hat{\mathbf{x}}$, and $\tilde{\mathbf{d}}$ to denote the perturbed demand vector, we write $\Upsilon_{w}$ using the first-order Taylor expansion:

$$
\Upsilon_{w}(\tilde{\mathbf{d}})=\hat{T}_{w}+\left\langle\nabla \hat{T}_{w}, \tilde{\mathbf{d}}-\hat{\mathbf{d}}\right\rangle
$$

where $\nabla \hat{T}_{w}$ is the $K$-dimensional gradient vector of $\hat{T}_{w}$ with respect to OD flows evaluated at $\hat{\mathbf{x}}$, and $\langle x, y\rangle$ is the inner product of vectors $x$ and $y$. In (5), it is assumed that the travel time between OD pair $w$ is a function of all demand vector entries. This assumption, however, requires estimating $\mathcal{O}\left(K^{2}\right)$ parameters which is not practically feasible. Hence we model the travel time between OD pair $w$ as a function of a subset of OD demands. Let $G(w)$ (of cardinality $g_{w}$ ) denote the set of OD demands selected to model the travel time of OD pair $w$ in the contracted network. Then, (5) can be written as:

$$
\Upsilon_{w}\left(\tilde{\mathbf{e}}_{\mathbf{w}}\right)=\hat{T}_{w}+\left\langle\boldsymbol{\psi}_{w}, \tilde{\mathbf{e}}_{w}-\hat{\mathbf{e}}_{w}\right\rangle
$$

where $\tilde{\mathbf{e}}_{w}, \hat{\mathbf{e}}_{w}$, and $\psi_{w}$ are, respectively, $g_{w}$-dimensional subvectors of vectors $\tilde{\mathbf{d}}, \hat{\mathbf{d}}$, and $\nabla \hat{T}_{w}$ corresponding to OD pairs in $G(w)$. A small example in Section 6 describes this model approximation in more detail.

In general, $g_{w}$ can be any number between 1 and $K$ where $g_{w}=1$ means that $\Upsilon_{w}$ is just a function of $d_{w}$ (the assumption by Boyles, 2012), and for $g_{w}=K, \Upsilon_{w}$ is a function of all OD flows. The value of $g_{w}$ is a trade-off between the accuracy of the contracted model and computational time required to estimate the contracted network, where a smaller $g_{w}$ requires estimating fewer parameters, but such a model may be prone to higher error rates since the effect of some OD demands are neglected. A heuristic for choosing this subset of OD pairs is provided below. 
In equation (6), the only unknowns to be estimated for OD pair $w$ are the $g_{w}$ components of the vector $\boldsymbol{\psi}_{w}$. The entry of the vector $\boldsymbol{\psi}_{w}$ associated with OD pair $u$, i.e. $\partial \hat{T}_{w} / \partial d_{u}$, shows the derivative of $\hat{T}_{w}$ with respect to $d_{u}$ evaluated at $\hat{\mathbf{x}}$. The theorem below shows that under the assumption that OD stems remain unchanged, these interactions are symmetric: a small change in $\hat{d}_{u}$ has the same impact on $\hat{T}_{w}$ as a small change in $\hat{d}_{w}$ would have on $\hat{T}_{u}$.

Theorem 1. For any two OD pairs $w$ and $u$ :

$$
\frac{\partial \hat{T}_{w}}{\partial d_{u}}=\frac{\partial \hat{T}_{u}}{\partial d_{w}}
$$

Proof. Tobin and Friesz (1988) showed that for a small demand perturbation, where the set of used paths remain intact, the change in equilibrium travel time would be:

$$
\partial \mathbf{T}=\left[\Lambda(\nabla \mathbf{C})^{-1} \Lambda^{t}\right]^{-1} \partial \mathbf{d}
$$

where $\partial \mathbf{T}$ and $\partial \mathbf{d}$ are, respectively, differential vectors of changes in OD travel time and demand, and $\nabla \mathbf{C}$ is Jacobian of path cost vector with respect to path flow:

$$
\nabla \mathbf{C}=\left[\begin{array}{cccc}
\frac{\partial C_{1}}{\partial h_{1}} & \frac{\partial C_{1}}{\partial h_{2}} & \ldots & \frac{\partial C_{1}}{\partial h_{\rho}} \\
\frac{\partial C_{2}}{\partial h_{1}} & \frac{\partial C_{2}}{\partial h_{2}} & \ldots & \frac{\partial C_{2}}{\partial h_{\rho}} \\
\vdots & \vdots & \ddots & \vdots \\
\frac{\partial C_{\rho}}{\partial h_{1}} & \frac{\partial C_{\rho}}{\partial h_{2}} & \cdots & \frac{\partial C_{\rho}}{\partial h_{\rho}}
\end{array}\right]
$$

where $\rho$ is the number of paths with positive demand, and $C_{\pi}$ is travel time on path $\pi . \Lambda$ is the OD/path incidence matrix with $K$ rows and $\rho$ columns and $\Lambda(w, \pi)=1$ if path $\pi$ is used by $d_{w}$, and 0 otherwise.

Noting that $\frac{\partial x_{a}}{\partial h_{\pi}}=1$ if link $a$ is utilized by path $\pi, \delta_{a \pi}=1$, for two paths $\pi$ and $\eta$ we have:

$$
\frac{\partial C_{\pi}}{\partial h_{\eta}}= \begin{cases}\sum_{a \in A} \frac{\partial t_{a}}{\partial x_{a}} \delta_{a \pi} & \text { if } \pi=\eta \\ \sum_{a \in A} \frac{\partial t_{a}}{\partial x_{a}} \delta_{a \pi} \delta_{a \eta} & \text { otherwise }\end{cases}
$$

Using this equality, we can see that for two different paths $\pi$ and $\eta$ with some common links:

$$
\frac{\partial C_{\pi}}{\partial h_{\eta}}=\frac{\partial C_{\eta}}{\partial h_{\pi}}
$$

indicating that $\nabla \mathbf{C}$ (and thus its inverse) are symmetric:

$$
(\nabla \mathbf{C})^{-1}=\left((\nabla \mathbf{C})^{-1}\right)^{t}
$$

We can thus show that $\Lambda(\nabla \mathbf{C})^{-1} \Lambda^{t}$ is symmetric:

$$
\left(\Lambda(\nabla \mathbf{C})^{-1} \Lambda^{t}\right)^{t}=\Lambda\left(\Lambda(\nabla \mathbf{C})^{-1}\right)^{t}=\Lambda\left((\nabla \mathbf{C})^{-1}\right)^{t} \Lambda^{t}=\Lambda(\nabla \mathbf{C})^{-1} \Lambda^{t}
$$


which implies that $\left(\Lambda(\nabla \mathbf{C})^{-1} \Lambda^{t}\right)^{-1}$ is also symmetric. Let

$$
\left(\Lambda(\nabla \mathbf{C})^{-1} \Lambda^{t}\right)^{-1}=\left[\begin{array}{cccc}
\gamma_{11} & \gamma_{12} & \ldots & \gamma_{1 K} \\
\gamma_{21} & \gamma_{22} & \ldots & \gamma_{2 K} \\
\vdots & \vdots & \ddots & \vdots \\
\gamma_{K 1} & \gamma_{K 2} & \ldots & \gamma_{K K}
\end{array}\right]
$$

then

$$
\left[\begin{array}{c}
\partial \hat{T}_{1} \\
\partial \hat{T}_{2} \\
\vdots \\
\partial \hat{T}_{K}
\end{array}\right]=\left[\begin{array}{cccc}
\gamma_{11} & \gamma_{12} & \ldots & \gamma_{1 K} \\
\gamma_{21} & \gamma_{22} & \ldots & \gamma_{2 K} \\
\vdots & \vdots & \ddots & \vdots \\
\gamma_{K 1} & \gamma_{K 2} & \ldots & \gamma_{K K}
\end{array}\right]\left[\begin{array}{c}
\partial d_{1} \\
\partial d_{2} \\
\vdots \\
\partial d_{K}
\end{array}\right]
$$

It can be verified easily that change in equilibrium cost of OD pair $w$ in response to a small change in demand of OD pair $u$ is:

$$
\frac{\partial \hat{T}_{w}}{\partial d_{u}}=\gamma_{w u}
$$

and the same way:

$$
\frac{\partial \hat{T}_{u}}{\partial d_{w}}=\gamma_{u w}
$$

these two values are equal.

Later, in 6.1, a numerical example on the small network described in Figure 2 clarifies this property and shows how to compute these derivatives and interactions.

This symmetry property can reduce the computational time required to estimate the contracted network by calculating just one derivative instead of doing two sensitivity analysis to compute $\partial \hat{T}_{u} / \partial d_{w}$ and $\partial \hat{T}_{w} / \partial d_{u}$. Consider a network with $K$ OD pairs and assume that we want to model the contracted network with full interactions. Furthermore, assume that all $K$ OD stems are of the same size. Without considering the symmetry property, we need to solve $K$ problems each with $K$ OD stems, a total of $K^{2}$ problems. With the symmetricity property, the problem size associated with the first OD pair is $K$, the second problem is $K-1$, and the last OD pair is 1 . The happens because after solving the sensitivity problem for OD pair $w_{1}$ and computing the derivative for OD pair $w_{2}, \partial \hat{T}_{w_{2}} / \partial d_{w_{1}}$, we do not need to include $\mathcal{B}_{w_{1}}$ when doing sensitivity analysis for OD pair $w_{2}$. This results in a total of $\frac{K(K-1)}{2}$ problems, roughly half the number needed without considering the symmetricity property.

From here on, the focus of the paper is the computation of these partial derivatives.

Assume that demand between OD pair $w$ is perturbed by a small value such that the set of equilibrium 
paths remain fixed, and the goal is to estimate the change in the travel time of OD pairs in $G(w)$. Under the assumption that OD stems remain unchanged, flow shifts between the stem paths of each OD pair $u \in G(w)$ until a new equilibrium is achieved and all the stem paths have the same cost. This indicates that travel time is changed by the same amount on all paths. The above condition can be stated as:

$$
\frac{\partial \hat{T}_{w}}{\partial d_{u}}=\frac{\partial \hat{T}_{u}}{\partial d_{w}}=\frac{\partial C_{\pi}}{\partial d_{w}}, \quad \forall \pi \in \hat{p}_{u}, u \in G(w)
$$

The changes in each path's travel time is equal to the sum of the changes in travel times of its links:

$$
\frac{\partial C_{\pi}}{\partial d_{w}}=\sum_{a \in A_{w}} \frac{\partial t_{a}}{\partial d_{w}} \delta_{a \pi}, \quad \forall \pi \in \hat{p}_{u}, u \in G(w)
$$

where $\partial t_{a} / \partial d_{w}$ is the derivative of link $a$ travel time with respect to $d_{w}$. Using the chain rule, the above equation can be written as:

$$
\frac{\partial C_{\pi}}{\partial d_{w}}=\sum_{a \in A_{w}} t_{a}^{\prime} \alpha_{a}^{w} \delta_{a \pi}, \quad \forall \pi \in \hat{p}_{u}, u \in G(w)
$$

where $t_{a}^{\prime}=d t_{a} / d x_{a}$ is the derivative of link travel time with respect to the link flow evaluated at $\hat{\mathbf{x}}$, and $\alpha_{a}^{w}=\partial x_{a} / \partial d_{w}$ is the derivative of link $a$ flow with respect to $d_{w}$ evaluated at $\hat{\mathbf{x}}$.

Let $\beta_{\pi}^{w}=\partial h_{\pi} / \partial d_{w}$ be the derivative of path $\pi$ flow with respect to $d_{w}$ and $\overline{\mathbf{A}}_{w}=\bigcup_{u \in G(w)} A_{u}$ be the union of the links on the stems of OD pairs in $G(w)$. Then we have:

$$
\alpha_{a}^{w}=\sum_{u \in G(w)} \sum_{\pi \in \hat{p}_{u}} \beta_{\pi}^{w} \delta_{a \pi}, \quad \forall a \in \overline{\mathbf{A}}_{w}
$$

In addition, since $d_{w}$ is the only independent variable, we can write:

$$
\sum_{\pi \in \hat{p}_{u}} \beta_{\pi}^{w}=\left\{\begin{array}{ll}
1 & w=u \\
0 & w \neq u
\end{array}, \quad \forall u \in G(w)\right.
$$

Equation (21) indicates that one unit of demand is added to $d_{w}$ and the demand between the remaining OD pairs remain unchanged. The linear system of equations described by (17), (19), (20), and (21) can be solved for $\beta_{\pi}^{w}$ and compute $\partial \hat{T}_{u} / \partial d_{w}$ for every OD pair $u \in G(w)$ which form the entries of $\boldsymbol{\psi}_{w}$. This procedure needs to be repeated for each OD pair $w \in W$.

The linear system (17), (19), (20), and (21) form the basis for the sensitivity analysis in this paper. Boyles (2012) describes two methods for calculating these derivatives. The first approach is based on network transformations and has similarities with techniques used in analysis of resistive circuits. Four network transformations are proposed: two series links to one link; two parallel link to one link; $\Delta$ structure (an undirected cycle of three nodes with an empty interior) to $\mathbf{Y}$, and $\mathbf{Y}$ structure to $\Delta$. These four transformations are illustrated in Figure 3. Under the planarity assumption, these transformations can be used to successively replace each OD stem with a simpler stem. At the end, the stem is reduced to a single arc, and OD derivative 

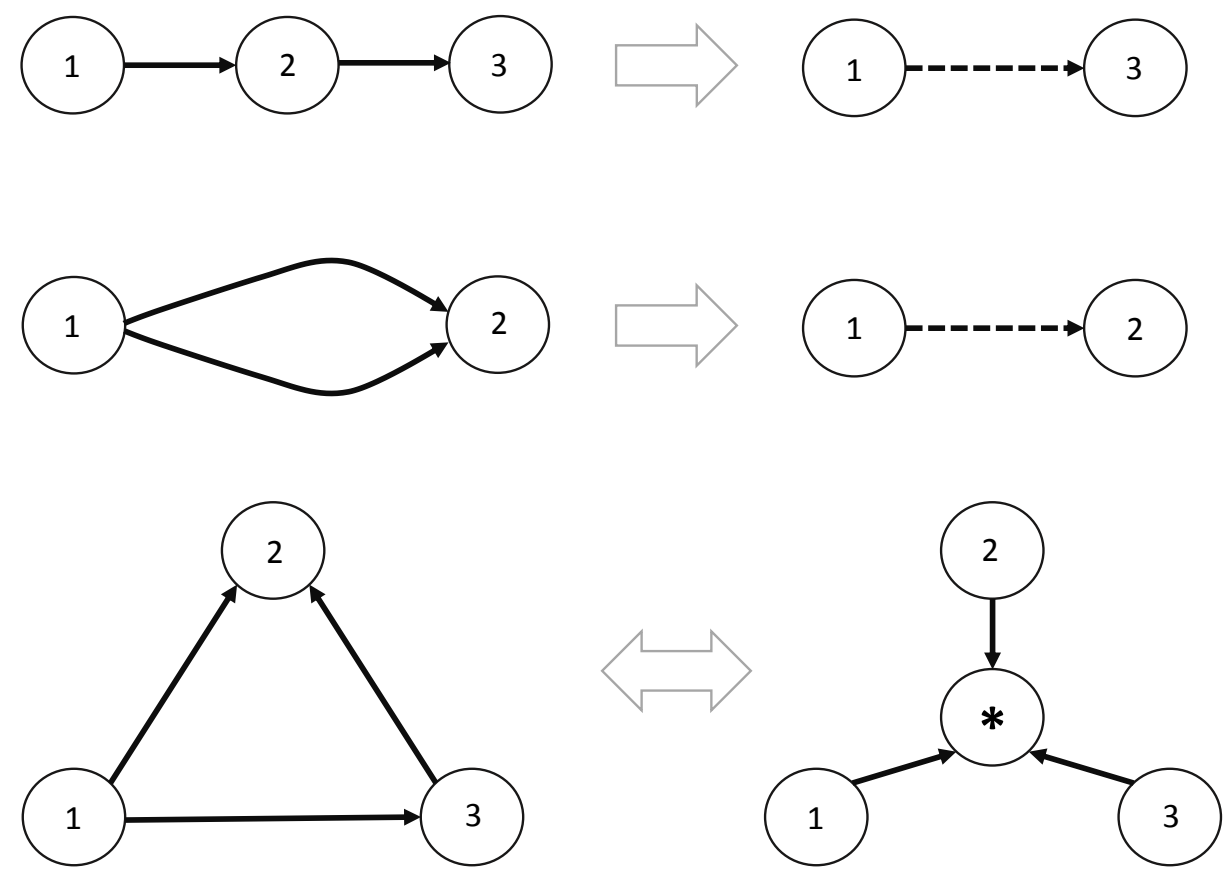

Figure 3: Schematic of series (top), parallel (middle), and $\Delta-\mathbf{Y}$ (bottom) transformations

can be easily calculated.

The second technique calculates these derivatives by directly solving equations (17), (19), (20), and (21) enforcing that the stem remains at equilibrium. The system of linear equation has $\mathcal{O}(n+m)$ equations and requires inverting a matrix of size $\mathcal{O}(n+m)$. Using a well-known block inversion formula, Boyles (2012) proposes a technique that iteratively solves this linear system of equations, ensuring that no matrix needs to be inverted with dimension larger than the number of incident arcs to a single node.

In summary, the first technique is limited to planar bushes, which is a restrictive assumption. The second technique is more general and does not require any planarity assumption, but has higher computational time and needs careful implementation to avoid numerical instabilities from repeated matrix reinversion. In the next section, however, we propose a faster solution method based on formulating this system as the solution to a convex program. 


\section{Equilibrium Formulation}

Rather than solving (17), (19), (20), and (21) directly as a linear system, in this section we show that the solution to this system also solves the convex optimization problem:

$$
\begin{array}{ll}
\operatorname{minimize} & \sum_{a \in \overline{\mathbf{A}}_{w}} \int_{0}^{\alpha_{a}^{w}} t_{a}^{\prime} \omega d \omega \\
\text { subject to } & \sum_{\pi \in \hat{p}_{u}} \beta_{\pi}^{w}=0, \quad \forall u \in G(w), u \neq w \\
& \sum_{\pi \in \hat{p}_{w}} \beta_{\pi}^{w}=1 \\
& \alpha_{a}^{w}=\sum_{u \in G(w)} \sum_{\pi \in \hat{p}_{u}} \beta_{\pi}^{w}, \quad \forall a \in \overline{\mathbf{A}}_{w}
\end{array}
$$

This is essentially a static equilibrium problem on the network comprised of all OD stems in $G(w)$, with $\alpha_{a}^{w}$ and $\beta_{\pi}^{w}$ serving the role of link flows and path flows, respectively, linear cost functions of the form $t_{a}^{\prime} \alpha_{a}^{u}$, and unit demand between OD pair $u$. There is one significant difference: there is no nonnegativity constraint on the $\beta_{\pi}^{u}$, reflecting the fact that not all path flows need increase with $d_{w}$. The Braess network (Braess, 1969) forms a counterexample: as the reader can verify, when the demand between the endpoints increases from 6, flow on the path utilizing the middle link decreases.

The objective function (22) is strictly convex, and the constraints (23)-(25) form a convex set, so a solution exists and is unique. Lagrangianizing the flow conservation constraints (23) and (24) with multipliers $\tau^{w}$, the first-order conditions are

$$
\begin{array}{lr}
\sum_{a \in A_{u}} t_{a}^{\prime} \alpha_{a}^{w} \delta_{a \pi}-\tau_{u}^{w}=0, & \forall \pi \in \hat{p}_{u}, u \in G(w) \\
\sum_{\pi \in \hat{p}_{u}} \beta_{\pi}^{w}=0, & \forall u \in G(w), u \neq w \\
\sum_{\pi \in \hat{p}_{w}} \beta_{\pi}^{w}=1 & \forall a \in \overline{\mathbf{A}}_{w} \\
\sum_{u \in G(w)} \sum_{\pi \in \hat{p}_{u}} \beta_{\pi}^{w}=\alpha_{a}^{w}, &
\end{array}
$$

with no complementarity conditions because there are no nonnegativity constraints on $\boldsymbol{\beta}$. Interpreting $\tau_{u}^{w}$ as $\partial \hat{T}_{u} / \partial d_{w}$, the system (26)-(29) is identical to (17), (19), (20), and (21).

This equivalence presents an easier method to identify the travel time derivatives needed to form cost functions on the artificial arcs of the contracted network, because the optimization problem (22)-(25) can be solved by making minor changes to bush-based algorithms for solving the traffic assignment problem.

The bush-based algorithms start from the shortest path tree for each origin and iterate between equilibrating and modifying the bush. At each iteration, the origin bush is fixed and the network is reduced to an acyclic network containing just the bush links and the assignment problem is restricted to this acyclic 
network. Bar-Gera (2002) and Dial (2006) prove that the equilibrium flow is attained after all bushes are equilibrated. The general procedure may be described as follows:

1. Initialize the origin bush by finding the shortest path tree for each origin and assign all flows to the origin bush.

2. Add new links to bush if travel time can be improved.

3. Solve the assignment problem over the acyclic network restricted to the bush.

4. Remove links with zero flow.

5. Stop if converged, otherwise go to 2 .

The interested reader is referred to Nie (2010) for an in-depth discussion on different bush-based algorithms and their computational power.

In a bush-based algorithm, the only change needed is to eliminate the zero-flow lower bound when equilibrating the bush, and skipping the bush updating steps since these links are fixed. Further, by solving this equilibrium problem to varying gap levels, one can more finely adapt the precision of the method to the computational resources available. As shown in the next section's computational results, it is not necessary to calculate these derivatives with a high level of accuracy, because other aspects of the approximation (e.g., assuming fixed bushes) tend to dominate the error in the overall procedure.

The optimization problem (22)-(25) is a special case of the quadratic approximations developed by Patriksson (2004) and Josefsson and Patriksson (2007), for the case where only the OD matrix is perturbed, link costs are separable, and there is no elasticity in demand. Patriksson (2004) obtained the model starting from results on the sensitivity of variational inequalities. This paper presents an alternative derivation starting from equilibrium and flow conservation principles, showing that these equations form the KKT conditions for the quadratic approximation model given in the paper. We believe that there is value in presenting such an alternative derivation, and that it may be more intuitive for some readers (albeit for a special case of Patriksson's results.) Also the problem (22)-(25) is disaggregated, only considering the interactions between partial sets of OD pairs. This allows the sensitivity subproblem to be separated by OD pair, which may have computational advantages (including easier parallelization).

In this section, a formulation similar to classical user equilibrium problem was proposed to compute the sensitivity of travel time for every OD pair $u \in G(w)$ in response to a small change in $d_{w}$. The question still remained to be answered is which OD pairs should be included in $G(w), \forall w \in W$. The next section will address this question by proposing a simple heuristic.

\section{Network Interactions}

It is important to consider how the list of OD flows for modeling the cost on $l_{w}$ in (6) is chosen for every $w \in W$. The goal of embedding the other flows in $\Upsilon_{w}$ is achieving an approximation with lower estimation error. Based on this, the list $G(w)$ for OD pair $w \in W$ should contain those OD pairs for which a small 
change in their flow has the highest impact on travel cost of $w$. Estimating the dependency of each OD travel time on other OD flows, however, requires doing sensitivity analysis for all OD pairs which is a computationally challenging and practically infeasible task. To alleviate this problem and still gain a good approximation, a simple heuristic is proposed here.

For each OD pair, the links with the highest sensitivity — defined as derivative of link travel time with respect to its flow - play the role of bottlenecks. For example, a link with derivative of 1 sec/vehicle has a small impact on route choice of users compared to a link with derivative of $10 \mathrm{sec} / \mathrm{vehicle}$. It is clear that a small change in flow of such bottleneck links may encourage some travelers to search for cheaper routes. This is related to the concept of intrinsic sensitivity, defined in Boyles et al. (2011).

Let $O D_{a}$ denote the list of OD pairs with demand on link $a$. Given $g_{w}$ for every OD pair $w \in W$, Algorithm 1 calculates the list $G(w)$ in the following way. First, the link travel time derivatives for all links with positive demand are calculated. Then all OD stems are checked and $w$ is added to the list $O D_{a}$ if link $a$ is part of the stem of OD pair $w$. These two steps are preprocessing steps, and can be implemented without any further computational effort while solving for the UE. Then for each OD pair $w \in W$, the first bottleneck link in $A_{w}$, the link with the highest sensitivity, is computed and the most dominant OD pair, defined as the OD pair with the highest share of flow on this bottleneck link, is added to $G(w)$. To make sure that the evaluated bottleneck link and selected OD pair are not considered again, they are removed from the associated lists. If the number of OD pairs inserted in $G(w)$ is less than $g_{w}$, the first bottleneck link among the present links in $A_{w}$ is selected and the same process is repeated by selecting the dominant OD pair and adding it to $G(w)$. This process stops when $g_{w}$ OD pairs are selected for OD pair $w$ or all links are evaluated where in the latter case, the cardinality of $G(w)$ can be less than $g_{w}$. The algorithm can easily be modified to make sure that the list $G(w)$ contains $g_{w}$ OD pairs by selecting more than one OD pair from each bottleneck link. This process is repeated until all OD pairs $w \in W$ are evaluated. Note that $g_{w}$ 's are the input parameters in this heuristic, and can vary by OD pair.

The next section is devoted to evaluate the performance and computational time of the approximate model under different demand and modeling scenarios. Later the advantage of the proposed network contraction technique is described in subnetwork analysis where areas outside the subnetwork boundary are replaced by some artificial links to capture the impact of subnetwork modifications on demand in areas beyond the subnetwork boundary.

\section{Demonstration}

In this section, three test networks are used to implement the idea presented in this paper. The first case study is the toy network sketched in Figure 2. This network can be solved manually and helps the reader to follow the discussion of the paper. Then, the Barcelona network is used to illustrate the quality of the contracted network in capturing the behavior of the complete network. Also the effect of $g_{w}$ on the accuracy of the model and time required for estimating the unknown parameters of the contracted network is evaluated.

Finally, the regional Austin, Texas network is used for the purpose of subnetwork analysis. All experiments are implemented in Java and carried out on a PC with an Intel Core i7 1.8GHz CPU and main memory of 


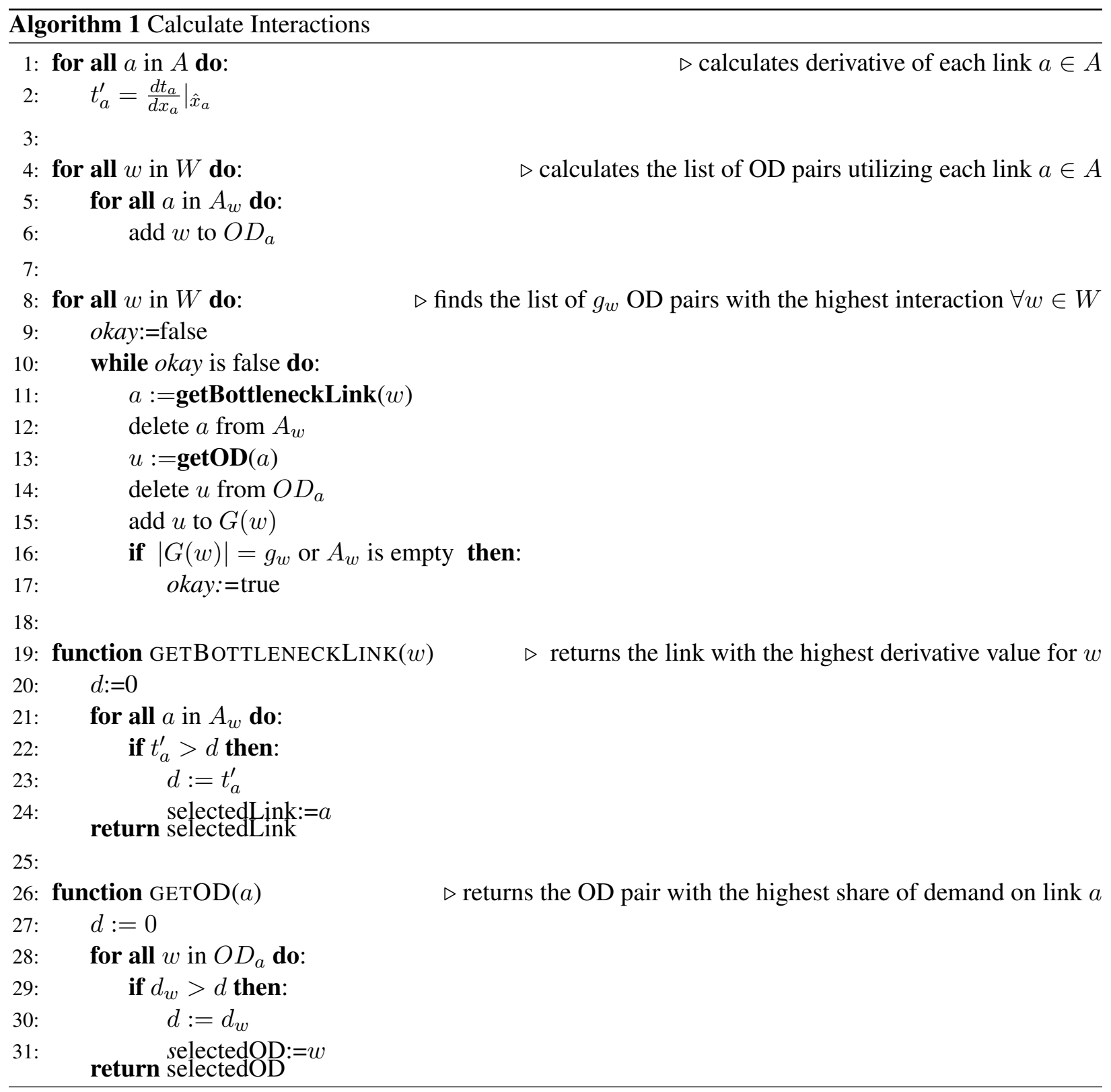


Table 2: The link cost functions of the network in Figure 2.

\begin{tabular}{l|ccccccc} 
Link & $1-2$ & $1-6$ & $4-3$ & $4-5$ & $6-5$ & $1-4$ & $2-3$ \\
\hline Cost function & 2 & 2 & 2 & 2 & $3 x_{15}$ & $x_{14}$ & $2 x_{23}$
\end{tabular}

4GB.

\subsection{Toy Network}

Consider the toy network in Figure 2 with link cost functions described in Table 2. Assume demand of $d_{15}$ and $d_{13}$ from origin node 1 to destination nodes 5 and 3 , respectively. At equilibrium, the path flows and OD travel times are as follows:

$$
\left\{\begin{array}{l}
\hat{h}_{1,2,3}=\frac{3}{11}\left(d_{13}+d_{15}\right) \\
\hat{h}_{1,4,3}=\frac{1}{11}\left(8 d_{13}-3 d_{15}\right) \\
\hat{T}_{13}=2+\frac{6}{11}\left(d_{13}+d_{15}\right) \\
\hat{h}_{1,6,5}=\frac{2}{11}\left(d_{15}+d_{13}\right) \\
\hat{h}_{1,4,5}=\frac{1}{11}\left(9 d_{15}-2 d_{13}\right) \\
\hat{T}_{15}=2+\frac{6}{11}\left(d_{15}+d_{13}\right)
\end{array}\right.
$$

Let $\tilde{d}_{13}$ and $\tilde{d}_{15}$ denote the perturbed demand values and $\hat{d}_{13}$ and $\hat{d}_{15}$ denote the current demands. According to equation (6), the cost of traveling on the artificial links in the contracted network, Figure 2 right panel, would be:

$$
\left\{\begin{aligned}
\Upsilon_{13}\left(\tilde{d}_{13}, \tilde{d}_{15}\right) & =\hat{T}_{13}+\frac{\partial T_{13}}{\partial d_{3}}\left(\tilde{d}_{13}-\hat{d}_{13}\right)+\frac{\partial T_{13}}{\partial d_{15}}\left(\tilde{d}_{15}-\hat{d}_{15}\right) \\
& =2+\frac{6}{11}\left(\hat{d}_{13}+\hat{d}_{15}\right)+\frac{6}{11}\left(\tilde{d}_{13}-\hat{d}_{13}\right)+\frac{6}{11}\left(\tilde{d}_{15}-\hat{d}_{15}\right) \\
& =2+\frac{6}{11}\left(\tilde{d}_{13}+\tilde{d}_{15}\right) \\
\Upsilon_{15}\left(\tilde{d}_{13}, \tilde{d}_{15}\right) & =\hat{T}_{15}+\frac{\partial T_{15}}{\partial d_{15}}\left(\tilde{d}_{15}-\hat{d}_{15}\right)+\frac{\partial T_{15}}{\partial d_{13}}\left(\tilde{d}_{13}-\hat{d}_{13}\right) \\
& =2+\frac{6}{11}\left(\hat{d}_{15}+\hat{d}_{13}\right)+\frac{6}{11}\left(\tilde{d}_{15}-\hat{d}_{15}\right)+\frac{6}{11}\left(\tilde{d}_{13}-\hat{d}_{13}\right) \\
& =2+\frac{6}{11}\left(\tilde{d}_{15}+\tilde{d}_{13}\right)
\end{aligned}\right.
$$

where $\Upsilon_{13}$ and $\Upsilon_{15}$ are approximate OD travel times formulated as a linear function of perturbed demand values $\tilde{d}_{13}$ and $\tilde{d}_{15}$. Since all link cost functions are linear, the linear approximates match the correct equations. Let $\Delta T=\tilde{T}-\hat{T}$ and $\Delta h=\tilde{h}-\hat{h}$ denote, respectively, the travel time and path flow deviations as a result of demand deviation $\Delta d=\tilde{d}-\hat{d}$. Then, relations between travel time, demand, and path flow 
deviations are as follows:

$$
\left\{\begin{array}{l}
\Delta T_{13}=\frac{6}{11}\left(\Delta d_{13}+\Delta d_{15}\right) \\
\Delta h_{1,2,3}=\frac{3}{11}\left(\Delta d_{13}+\Delta d_{15}\right)=\frac{\Delta T_{13}}{2} \\
\Delta h_{1,4,3}=\frac{1}{11}\left(8 \Delta d_{13}-3 \Delta d_{15}\right)=\Delta d_{13}-\frac{\Delta T_{13}}{2} \\
\Delta T_{15}=\frac{6}{11}\left(\Delta d_{13}+\Delta d_{15}\right) \\
\Delta h_{1,6,5}=\frac{2}{11}\left(\Delta d_{13}+\Delta d_{15}\right)=\frac{\Delta T_{15}}{3} \\
\Delta h_{1,4,5}=\frac{1}{11}\left(-2 \Delta d_{13}+9 \Delta d_{15}\right)=\Delta d_{15}-\frac{\Delta T_{15}}{3}
\end{array}\right.
$$

These equations can be used easily to compute the amount of change in demand for each OD pair that results in a change $\Delta T$ in equilibrium travel times. For example, if the demand from 1 to 3 remains constant, i.e. $\Delta d_{13}=0$, then $\Delta h_{1,4,3}=-\Delta T / 2 ; \Delta h_{1,4,5}=3 \Delta T / 2$; and $\Delta d_{1,5}=11 \Delta T / 6$. The same way, if the demand from 1 to 5 remains constant, i.e. $\Delta d_{15}=0$, then $\Delta h_{1,4,5}=-\Delta T / 3 ; \Delta h_{1,4,3}=4 \Delta T / 3$; and $\Delta d_{1,3}=11 \Delta T / 6$.

Note that the equations in (31) are estimated with full interactions, and the OD travel times where interactions are not modeled would be as follows:

$$
\left\{\frac{\Upsilon_{13}\left(\tilde{d}_{13}\right)=2+\frac{2}{3} \tilde{d}_{13}}{\Upsilon_{15}\left(\tilde{d}_{15}\right)=2+\frac{3}{4} \tilde{d}_{15}}\right.
$$

which is not as accurate as the case with full interactions. The travel time derivatives in equations (31) and (33) can easily be computed by solving the optimization problem (22)-(25).

\subsection{Barcelona}

The method presented in the previous section is applied to Barcelona network with 110 zone, 1020 nodes, and 2522 links (Bar-Gera, 2013). As stated before, the travel cost between each artificial link $l_{w}$ in the contracted network is a linear approximation of the travel cost between OD pair $w$ in the complete network.

The complete network is solved to relative gap of $10^{-6}$ defined as:

$$
\text { relative gap }=\frac{\sum_{w \in W} \sum_{\pi \in \hat{p}_{w}} h_{\pi} C_{\pi}-\sum_{w \in W} \sum_{\pi \in \hat{p}_{w}} h_{\pi} \kappa^{w}}{\sum_{w \in W} \sum_{\pi \in \hat{p}_{w}} h_{\pi} C_{\pi}}
$$

where $\kappa^{w}$ represents the time spent on the fastest path between OD pair $w$. As verified by Boyce et al. (2004), this relative gap is enough to ensure that traffic assignment is converged to a stable link flow solution.

The equilibrium formulation (22)-(25) is used to compute the OD travel time derivatives with respect to OD demands. The equilibrium travel times along with these derivatives are needed to set up the contracted network. To evaluate the accuracy of the proposed network approximation algorithm, the OD matrix is perturbed, and the new equilibrium travel times are compared against those estimated via (6). For convenience, it is assumed that all OD pairs are modeled with the same number of OD interactions, i.e. $g_{w}=g$. Figure 4 shows the simulation results for different $g$ values and demand scenarios. Each line shows one demand sce- 
nario where $p \%$ means that all OD demands are perturbed randomly by $p$ percentage from the base values. The horizontal axis represents the number of OD flows selected to model the travel cost of each OD pair, $g$, and the vertical axis shows the average error between the actual travel time in the complete network and estimated travel time calculated from the contracted network. The estimation error is this figure is calculated as:

$$
\epsilon=\frac{1}{K} \sum_{w \in W} \frac{\left|\Upsilon_{w}(\tilde{\mathbf{e}})-\hat{T}_{w}(\tilde{\mathbf{d}})\right|}{\hat{T}_{w}(\tilde{\mathbf{d}})}
$$

where $\epsilon$ is the average estimation error per OD pair, $\tilde{\mathbf{d}}$ is the perturbed demand vector, and $\tilde{\mathbf{e}}$ is a subset of $\tilde{\mathbf{d}}$ corresponding to the entries of $G(w) . \Upsilon_{w}(\tilde{\mathbf{e}})$ is the estimated travel time between OD pair $w$ using the contracted network for perturbed demand $\tilde{\mathbf{d}}$, and $\hat{T}_{w}(\tilde{\mathbf{d}})$ is the actual travel time obtained by solving the complete network for $\tilde{\mathbf{d}}$.

As expected, the contracted network yields better results for demand scenarios with lower perturbation, and larger demand perturbations produces higher errors. This is due to the fact that the approximate model is a first order Taylor series calibrated for base demand and as demand deviates more from the calibration point, the accuracy of the model deteriorates. Also under each demand scenario, including more OD flows to model the travel time of each OD pair provides more accurate travel time estimates where improvement is more significant for a larger perturbation. For example, under $50 \%$ perturbation scenario, the error of $6.7 \%$ for the case where OD travel time is modeled as a function of only its own demand drops to $3.6 \%$ when 200 other OD flows are also included to model the interactions; an improvement of $3.1 \%$. The difference for perturbation of $5 \%$, however, is less than $0.4 \%$. This indicates that for a larger demand disruption, the impact of $g$ is more significant, but nevertheless the improvement is marginal, especially for small demand perturbations, and diminishes after $g \simeq 10$. Based on these observations, modeling OD travel times based on their own demand should be enough for most applications.

Figure 5 shows the time required to approximate and set up the contracted network. The horizontal axis represents dimension of gradient vector in (6), $g$, and vertical axis shows the ratio of time required to set up the contracted network — computing the list of OD flows for modeling each OD pair based on Algorithm 1 and solving the convex program (22)-(25) for each OD pair - to the time required for solving the complete network. It can be seen that computational complexity of the problem is affected by $g$, because it takes more time to set up the contracted network for a larger $g$ value. This is mainly because modeling more interactions increases the size of the problem in (22)-(25): for $g=1$, we need to solve these system of equations for one stem, while modeling all interactions requires solving a problem with size of the complete network for each OD pair. The results described in Figure 5 show that in case of Barcelona network with $g \leq 150$, it takes less time to set up the contracted network than solving the complete network.

The next section illustrates the benefits of the proposed network contraction technique for subnetwork analysis where the network outside the subnetwork boundary is replaced with an aggregated version to reduce the computational time and still capture the important attraction and diversion effects as drivers (globally) change routes in response to (local) subnetwork changes. 


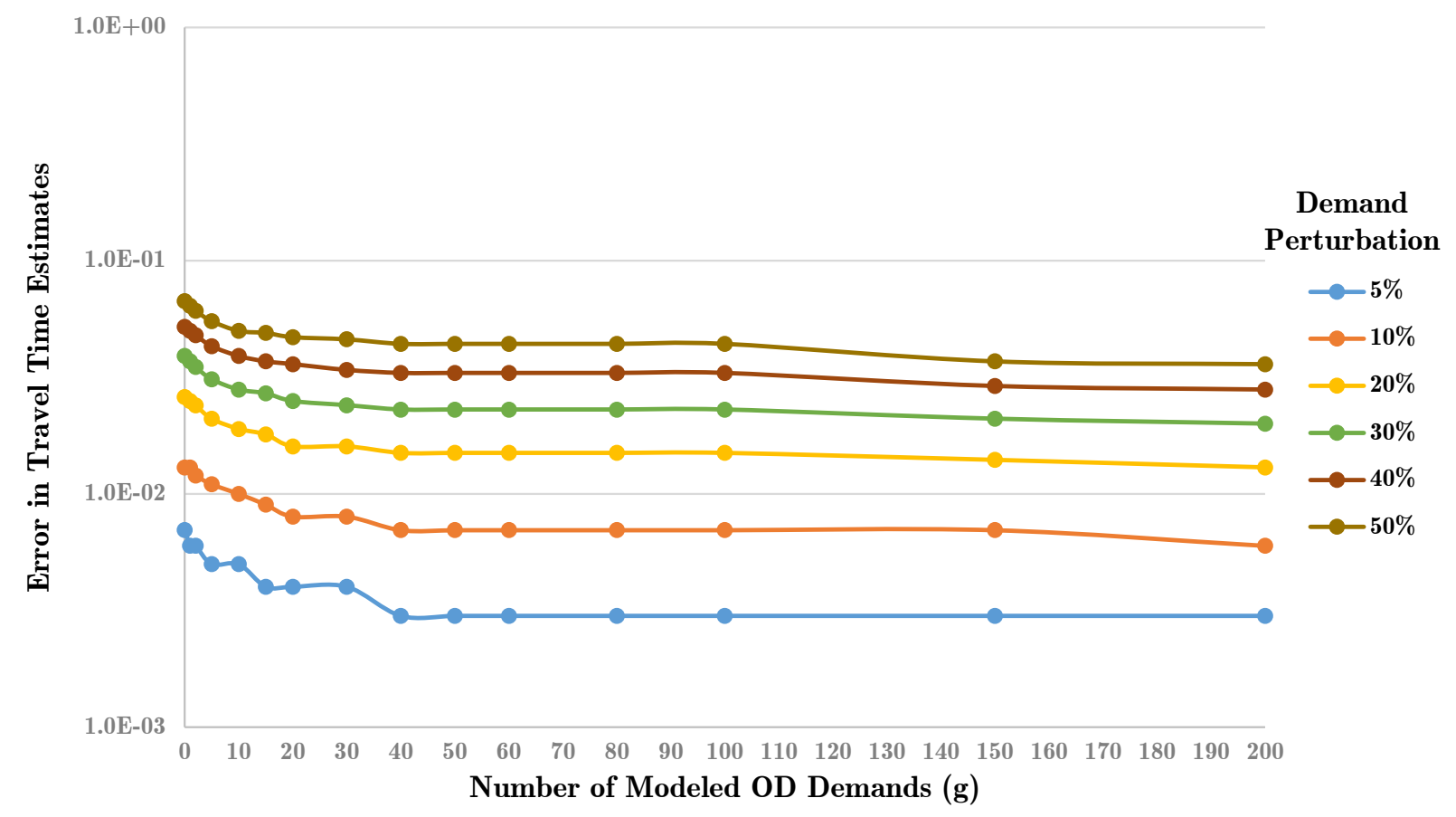

Figure 4: Total error between the travel times of the complete network and contracted network under different demand perturbation scenarios.

\subsection{Subnetwork Application}

To enable direct comparison with the methods developed by Boyles (2012), the same experimental setting was adopted. The regional network represents the Austin, TX metropolitan area, and contains 7466 nodes; 18,718 links; and 1117 zones; the downtown subnetwork contains 143 nodes, 448 arcs, and 20 internal zones (Figure 6). In the original network, 7th Street is a downtown arterial which is one-way eastbound.

We consider the impact of converting 7th Street to two ways, dividing its capacity equally between the two directions, and compare three techniques for evaluating the performance of the modified network. The first technique is to simply re-solve the equilibrium problem on the entire regional network; as reported in Boyles (2012), this requires approximately 20 minutes to reduce the relative gap to the range $10^{-4}$ $10^{-6}$, according to current recommendations Boyce et al. (2004). The second technique is to solve the equilibrium problem on the subnetwork alone, using the route flow solution from the base case network to form the OD matrix between boundary nodes; this requires only a second or two of computation time. The third technique is based on network contraction for $g_{w}=1$ by first creating artificial arcs as discussed in Section 3, then eliminating artificial arcs directly connecting an origin to a destination by introducing elastic demand and inverting the Gartner transformation (Boyles, 2012). Since the equilibrium bushes are nonplanar, the artificial arc cost functions can be found either by solving linear system of the form (17), (19), (20), and (21) (which requires approximately 90 minutes), or by solving modified equilibrium problem of the form (22)-(25). Either method gives identical results when the equilibrium problems are solved to a very tight gap, so the focus on this section is comparing the computational requirements involved, and the 


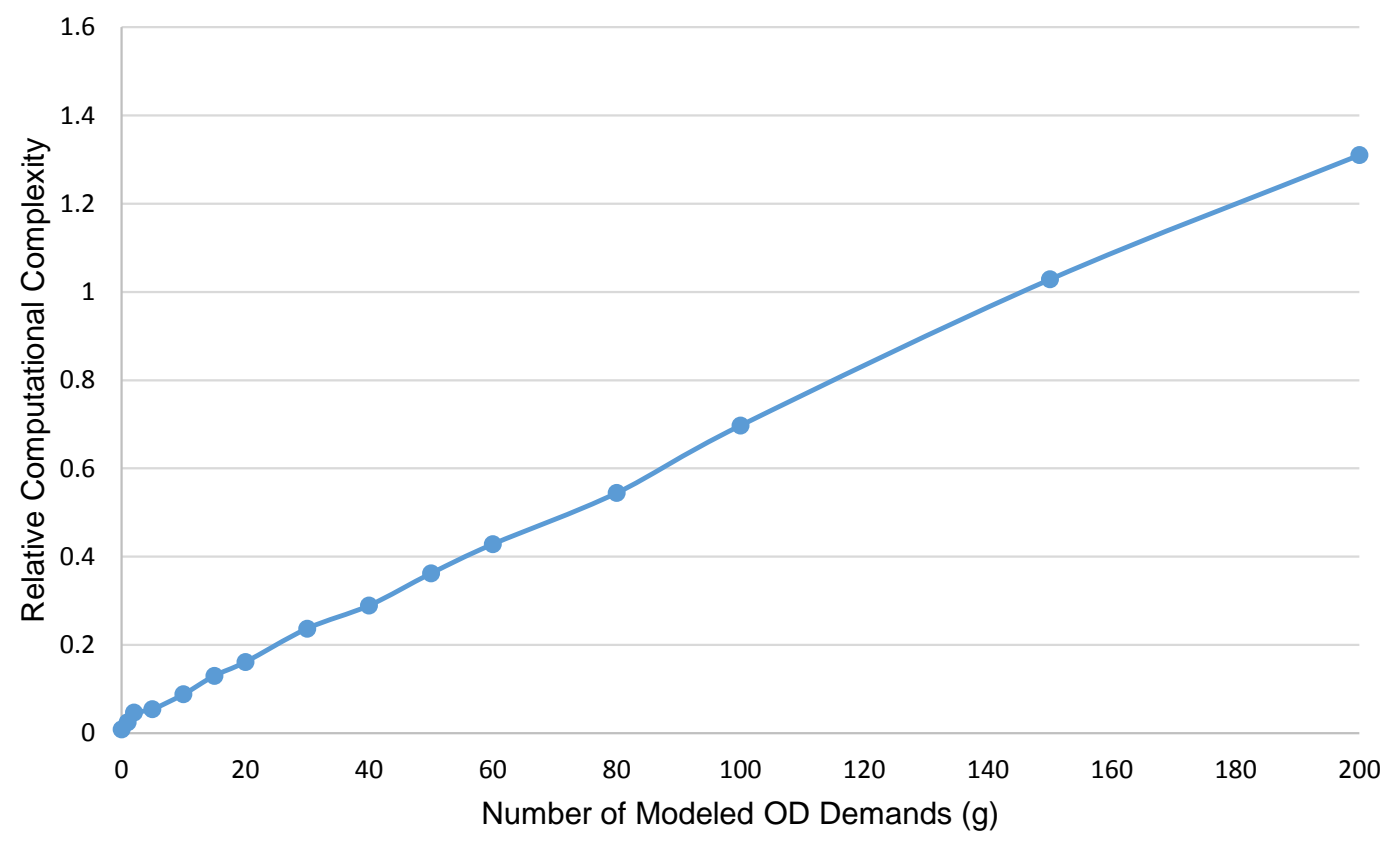

Figure 5: Ratio of time required to set up the contracted network to time of solving the complete network

tradeoffs between accuracy and computational time.

The remainder of this section investigates three main questions: first, the computation time required to solve (22)-(25) to varying gap levels; second, how the relative gap used in the equilibrium problems in the third technique affects the accuracy of the approximation; and third, how the relative gap used in the elastic demand subnetwork problem in the third technique affects the accuracy of the approximation. This accuracy is measured in terms of link volumes, link travel times, and corridor travel times in each direction.

Each equilibrium problem (22)-(25) can be solved very rapidly, in far less than a second. However, creating the contracted network requires solving 212,432 such problems. Figure 7 shows how the total computation time required varies with the relative gap criterion used to terminate the equilibrium problems. Solving the equilibrium subproblems to a relative gap of $10^{-4}$ requires roughly an order of magnitude less time than solving the linear systems directly. One may question the usefulness of this procedure, since the full regional network can be solved in only a little more time than is required to construct the approximation. The advantage comes in network design or other problems where subnetwork equilibrium must be solved many times, since the overhead involved in calculating these derivatives is only incurred once.

Table 3 shows how the root-mean square error (RMSE) link volume varies according to the relative gap used to calculate travel time derivatives, and the relative gap used when solving the elastic-demand equilibrium problem on the contracted network. These RMSE errors are calculated relative to the equilibrium solution on the regional network which serves as a baseline. By comparison, the RMSE from the fixed-boundary flows approach (the second technique) is $1216 \mathrm{vph}$. Notice that the accuracy of the third technique is not particularly sensitive to the gap values used. This indicates that the primary source of error in the approximation is due to the central assumptions made (fixed bushes and fixed flows outside 


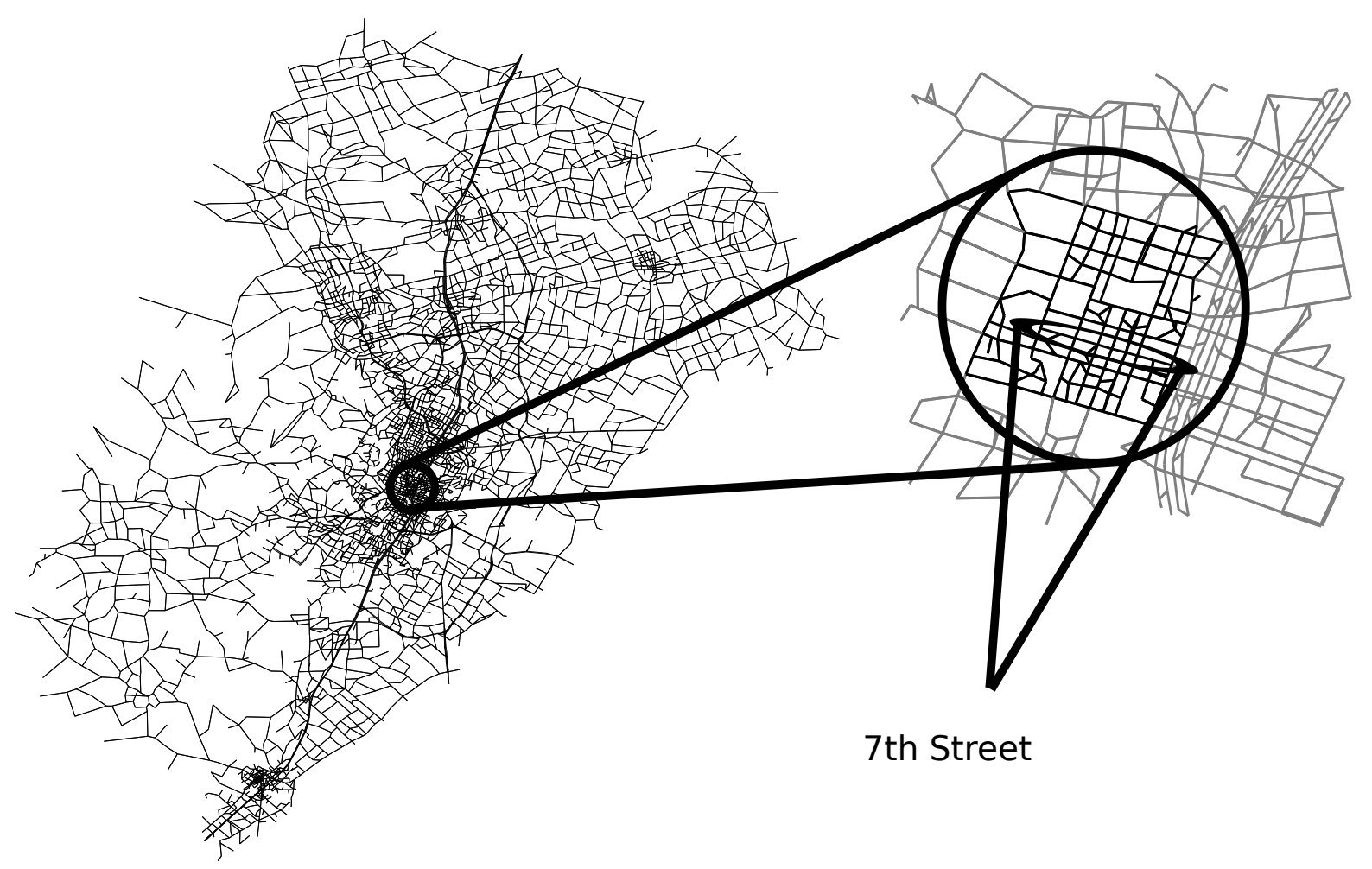

Figure 6: Austin regional network, subnetwork, and street modified from one-way to two-way.

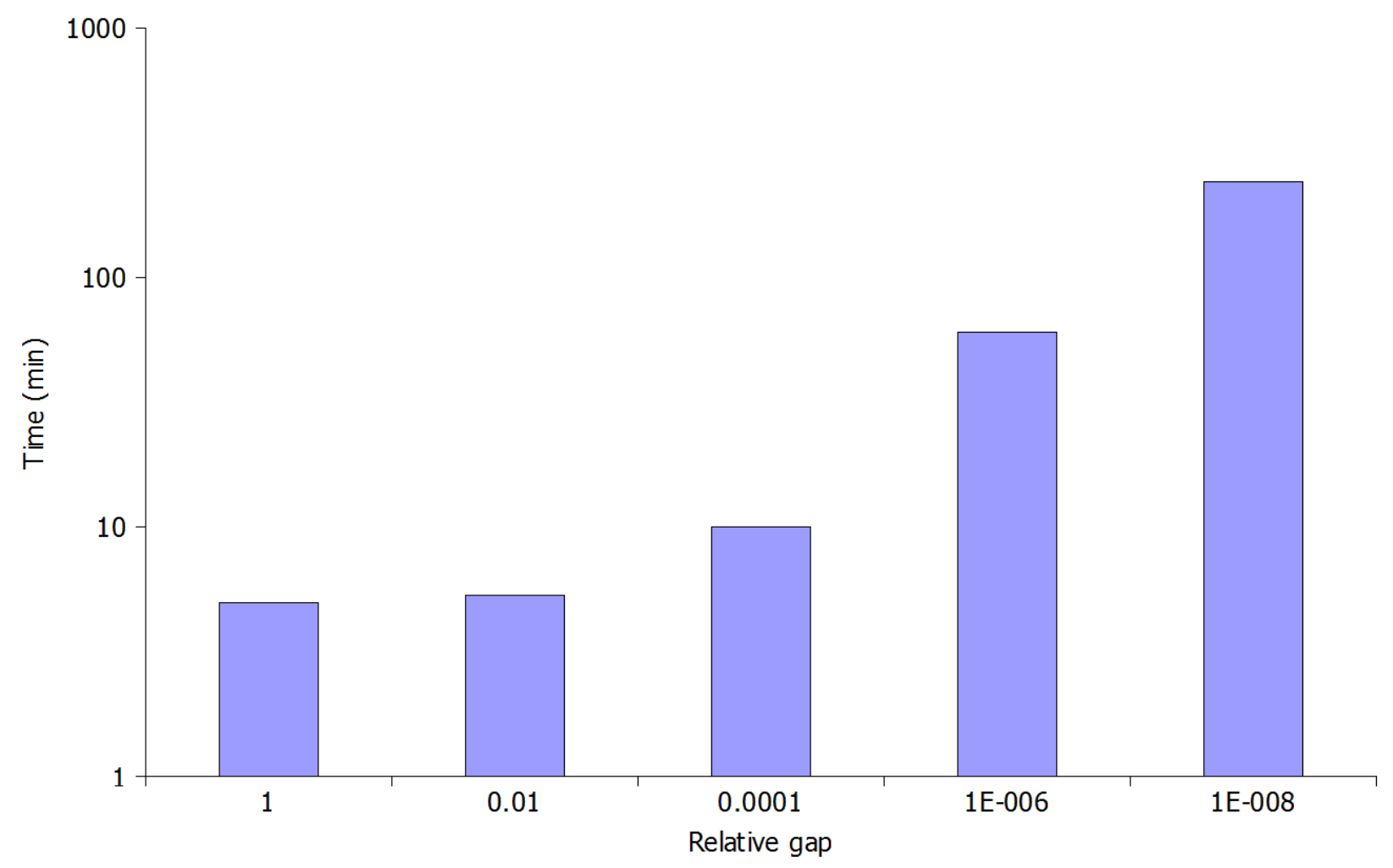

Figure 7: Computation time required for solving 212,432 modified equilibrium problems. 
Table 3: Link-flow RMSE for contracted graph as subproblem accuracy varies.

\begin{tabular}{rc|cccc} 
& & \multicolumn{4}{|c}{ Relative gap for solving contracted graph } \\
& & $10^{-2}$ & $10^{-4}$ & $10^{-6}$ & $10^{-8}$ \\
\hline Relative gap & 1 & 791.150 & 778.596 & 778.596 & 778.536 \\
for generating & $10^{-2}$ & 791.102 & 795.802 & 778.665 & 778.530 \\
contracted & $10^{-4}$ & 791.104 & 793.825 & 778.758 & 778.530 \\
graph & $10^{-6}$ & 791.112 & 795.228 & 778.600 & 778.529
\end{tabular}

Table 4: Average corridor travel time error for contracted graph as subproblem accuracy varies.

\begin{tabular}{rc|cccc} 
& & \multicolumn{5}{|c}{ Relative gap for solving contracted graph } \\
& & $10^{-2}$ & $10^{-4}$ & $10^{-6}$ & $10^{-8}$ \\
\hline Relative gap & 1 & $-3.471 \%$ & $-3.235 \%$ & $-3.235 \%$ & $-3.231 \%$ \\
for generating & $10^{-2}$ & $-5.817 \%$ & $-5.982 \%$ & $-5.439 \%$ & $-5.435 \%$ \\
contracted & $10^{-4}$ & $-5.814 \%$ & $-5.982 \%$ & $-5.437 \%$ & $-5.435 \%$ \\
graph & $10^{-6}$ & $-5.814 \%$ & $-5.976 \%$ & $-5.441 \%$ & $-5.435 \%$
\end{tabular}

each stem), rather than the accuracy to which the subproblems are solved. This result favors the method developed in this paper, because there is no need to calculate the travel time derivatives exactly. Table 4 shows similar results when comparing average corridor travel times (in both directions, the contracted graph slightly underestimated the travel times).

\section{Conclusion}

This paper presented a new method for calculating travel time sensitivities on equilibrium bushes which is superior to the two presented in earlier work by Boyles (2012): it can model interactions between different OD pairs, it requires no planarity assumption, is more stable numerically, is easier to implement using existing code for traffic assignment, and the computation time can be controlled by adjusting a gap termination criterion. Further, the simulation results of Barcelona network indicate that the contracted network can approximate the behavior of the complete network: the error is less that $6.7 \%$ even for a demand perturbation as high as $50 \%$ and can be reduced to $3.6 \%$ by modeling the interaction between OD travel times and OD flows. The application of the proposed approach for subnetwork analysis on the Austin, TX regional network shows that this method achieves comparable accuracy to the other methods while requiring only a tenth of the computation time. It would be useful to validate this performance on other networks, or to develop tailored network design or second-best pricing algorithms based on these approximations.

It should be noted that the contracted network only has to be constructed once and can be used to evaluate the network performance for different demand scenarios easily with a good approximation. The hybrid approach can be used to improve the accuracy of the model in a bi-level network design problem where hundreds or thousands of equilibrium subproblems are required to be solved. In the hybrid approach, contracted network is approximated based on the current demand and is fixed. After several iterations of working with the contracted network, it is updated by re-solving the complete network for the new demand vector. This reduces the estimation error by increasing the consistency between the contracted network and 
the complete network.

\section{Acknowledgments}

This material is based upon work supported by the National Science Foundation under Grant No. 1254921. Partial support was also provided by the Data-Supported Transportation Planning and Operations University Transportation Center. The authors would like to thank two anonymous referees for providing valuable suggestions on the manuscript which have improved its clarity and precision, including suggesting the example network in Figure 2.

\section{References}

R. K. Ahuja, T. L. Magnanti, and J. B. Orlin. Network Flows. Englewood Cliffs, NJ: Prentice-Hall, 1993.

H. Bar-Gera. Origin-based algorithm for the traffic assignment problem. Transportation Science, 36(4): 198-417, 2002.

H. Bar-Gera. Primal method for determining the most likely route flows in large road networks. Transportation Science, 40(3):269-286, 2006.

H. Bar-Gera. Traffic assignment by paired alternative segments. Transportation Research Part B: Methodological, 44(8):1022-1046, 2010.

H. Bar-Gera. Transportation test problems, April 2013. URL http: / /www . bgu • ac . i l/ bargera / tntp/.

H. Bar-Gera, F. Hellman, and M. Patriksson. Computational precision of traffic equilibria sensitivities in automatic network design and road pricing. Transportation Research Part B: Methodological, 57(4): 485-500, 2013.

P. H. L. Bovy and G. R. M. Jansen. Network aggregation effects upon equilibrium assignment outcomes. Transportation Science, 17(3):240-262, 1983.

D. Boyce, K. S. Chon, M. E. Ferris, Y. J. Lee, K. T. Lin, and R. W. Eash. Implementation and evaluation of combined models of urban travel and location on a sketch planning network. Technical report, University of Illinois at Urbana-mpaign, 1985.

D. Boyce, B. Ralevic-Dekic, and H. Bar-Gera. Convergence of traffic assignments: how much is enough? Journal of Transportation Engineering, 130(1):49-55, 2004.

S. D. Boyles. Bush-based sensitivity analysis for approximating subnetwork diversion. Transportation Research Part B: Methodological, 46(1):139-155, 2012. 
S. D. Boyles, A. Voruganti, and S. Waller. Quantifying distributions of freeway operational metrics. Transportation Letters, 3(1):21-36, 2011.

D. Braess. Uber ein Paradoxon aus der Verkehrsplanung. Unternehmensforschung, 12(1):258-268, 1969.

Y. Chan. A method to simplify network representation in transportation planning. Transportation Research, 10(3):179-191, 1976.

K. Chang, Z. Khatib, and Y. Ou. Effects of zoning structure and network detail on traffic demand modeling. Environment and Planning B, 29(1):37-52, 2002.

H. Cho, T. E. Smith, and T. L. Friesz. A reduction method for local sensitivity analyses of network equilibrium arc flows. Transportation Research Part B: Methodological, 34(1):31-51, 2000.

R. D. Connors and D. P. Watling. Assessing the demand vulnerability of equilibrium traffic networks via network aggregation. Networks and Spatial Economics, pages 1-29, 2014.

R. B. Dial. Probabilistic assignment: A multipath traffic assignment model which obviates path enumeration. PhD thesis, University of Washington, Seattle, Washington, 1970.

R. B. Dial. Minimal-revenue congestion pricing part I: A fast algorithm for the single-origin case. Transportation Research Part B: Methodological, 33(3):189-202, 1999a.

R. B. Dial. Accurate traffic equilibrium: how to bobtail frank-wolfe. Technical report, Volpe National Transportation Research Center, Cambridge, MA, 1999 b.

R. B. Dial. A path-based user-equilibrium traffic assignment algorithm that obviates path storage and enumeration. Transportation Research Part B: Methodological, 40(10):917-936, 2006.

R. W. Eash, K. S. Chon, Y. J. Lee, and D. Boyce. Equilibrium traffic assignment on an aggregated highway network for sketch planning. Transportation Research Record, 944:30-37, 1983.

T. L. Friesz. Transportation network equilibrium, design and aggregation: key developments and research opportunities. Transportation Research Part A: General, 19(5):413-427, 1985.

T. L. Friesz, R. L. Tobin, H.-J. Cho, and N. J. Mehta. Sensitivity analysis based heuristic algorithms for mathematical programs with variational inequality constraints. Mathematical Programming, 48(1-3): 265-284, 1990.

R. G. Gallager. A minimum delay routing algorithm using distributed computation. Communications, IEEE Transactions on, 25(1):73-85, 1977.

G. Gentile. Local user cost equilibrium: a bush-based algorithm for traffic assignment. Transportmetrica A: Transport Science, 10(1):15-54, 2014.

A. E. Haghani and M. S. Daskin. Network design application of an extraction algorithm for network aggregation. Transportation Research Record, 944:37-46, 1983. 
D. W. Hearn. Practical and theoretical aspects of aggregation problems in transportation planning. Transportation Planning Models, pages 257-287, 1984.

E. Jafari, M. Gemar, N. Ruiz-Juri, and J. Duthiel. An investigation of centroid connector placement for advanced traffic assignment models with added network detail. Accepted for Publication in Transportation Research Record: Journal of the Transportation Research Board, 2015.

J. Jeon, S.-Y. Kho, D.-K. Kim, and J.-S. Lee. Interactions of aggregated zoning and network systems: A case study of seoul city. Journal of the Eastern Asia Society for Transportation Studies, 8:404-419, 2010.

M. Josefsson and M. Patriksson. Sensitivity analysis of separable traffic equilibrium equilibria with application to bilevel optimization in network design. Transportation Research Part B: Methodological, 41(1): 4-31, 2007.

S. Lu. Sensitivity of static traffic user equilibria with perturbations in arc cost function and travel demand. Transportation science, 42(1):105-123, 2008.

S. Lu and Y. Nie. Stability of user-equilibrium route flow solutions for the traffic assignment problem. Transportation Research Part B: Methodological, 44(4):609-617, 2010.

Y. Nie. A class of bush-based algorithms for the traffic assignment problem. Transportation Research Part B: Methodological, 44(1):73-89, 2010.

M. Patriksson. Sensitivity analysis of traffic equilibria. Transportation Science, 38(3):258-281, 2004.

Y. Qiu and T. L. Magnanti. Sensitivity analysis for variational inequalities defined on polyhedral sets. Mathematics of Operations Research, 14(3):410-432, 1989.

H. Sbayti, M. El-Fadel, and I. Kaysi. Effect of roadway network aggregation levels on modeling of trafficinduced emission inventories in beirut. Transportation Research Part D: Transport and Environment, 7 (3):163-173, 2002.

R. L. Tobin and T. L. Friesz. Sensitivity analysis for equilibrium network flow. Transportation Science, 22 (4):242-250, 1988.

I. Wright, Y. Xiang, L. Waller, J. Cross, E. Norton, and D. Van Vliet. The practical benefits of the saturn origin-based assignment algorithm and network aggregation techniques. European Transport Conference, 2010.

C. Xie, K. M. Kockelman, and S. T. Waller. Maximum entropy method for subnetwork origin-destination trip matrix estimation. Transportation Research Record: Journal of the Transportation Research Board, 2196(1):111-119, 2010.

H. Yang and M. G. Bell. Sensitivity analysis of network traffic equilibrium revisited: The corrected approach. Mathematics in Transport (B. Heydecker, ed.), pages 373-411, 2007. 
N. D. Yen. Lipschitz continuity of solutions of variational inequalities with a parametric polyhedral constraint. Mathematics of Operations Research, 20(3):695-708, 1995.

P. H. Zipkin. Bounds for aggregating nodes in network problems. Mathematical programming, 19(1): $155-177,1980$. 